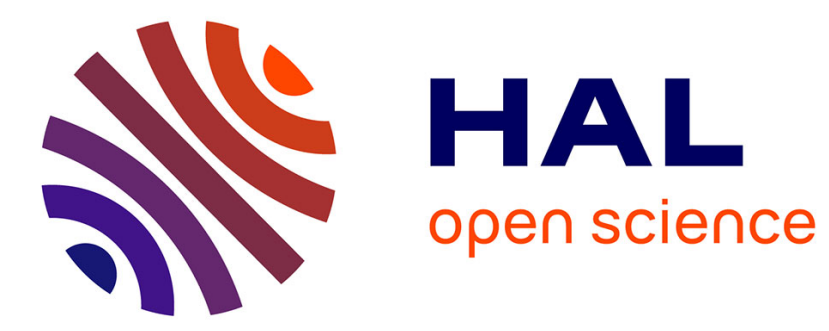

\title{
Argon 3s autoionization
}

S. Sorensen, T. Åberg, J. Tulkki, E. Rachlew-Källne, G. Sundström, M. Kirm

\section{To cite this version:}

S. Sorensen, T. Åberg, J. Tulkki, E. Rachlew-Källne, G. Sundström, et al.. Argon 3s autoionization. Journal de Physique IV Proceedings, 1994, 04 (C9), pp.C9-401-C9-404. 10.1051/jp4:1994967 . jpa00253530

\section{HAL Id: jpa-00253530 https://hal.science/jpa-00253530}

Submitted on 1 Jan 1994

HAL is a multi-disciplinary open access archive for the deposit and dissemination of scientific research documents, whether they are published or not. The documents may come from teaching and research institutions in France or abroad, or from public or private research centers.
L'archive ouverte pluridisciplinaire HAL, est destinée au dépôt et à la diffusion de documents scientifiques de niveau recherche, publiés ou non, émanant des établissements d'enseignement et de recherche français ou étrangers, des laboratoires publics ou privés. 


\title{
Argon 3s autoionization
}

\author{
S.L. Sorensen, T. Åberg*, J. Tulkki**, E. Rachlew-Källne, G. Sundström and M. Kirm*** \\ Department of Physics I, Royal Institute of Technology, 10044 Stockholm, Sweden \\ * Laboratory of Physics, Helsinki University of Technology, 02150 Espoo, Finland \\ ** Optoelectronics Laboratory, Helsinki University of Technology, 02150 Espoo, Finland \\ *** Department of Atomic Spectroscopy, University of Lund, 22362 Lund, Sweden
}

\begin{abstract}
Synchrotron radiation-excited ion mass spectrometry has been used to study the $3 s 3 p^{6} n p(n \geq 4)$ resonances in argon at $25.0-29.3 \mathrm{eV}$. It is shown using a time-independent resonant scattering approach that the Fano formalism may be applied to overlapping resonances. For the DubauSeaton profile an exact expression of the closed-channel phase shift is obtained. We find that regarding the window character of the Ar 3s series the Fano parameters calculated using the multichannel multiconfiguration Dirac-Fock method are in good agreement with those derived from fitting a convoluted Fano function to the measured $n=4$ autoionizing resonance. The Fano parameters are also derived from fits to the $5 \leq n \leq 8$ resonances which tests the validity of this parametrization to overlapping members of an inner-valence electron resonance series. In contrast, a parametrization of the entire series based upon the Dubau-Seaton formula did not result in a good fit without modifications. Using the same value of the shape parameter we find, however, that the $\mathrm{n}=4$ resonance can be accurately represented by both the Fano and Dubau-Seaton profiles.
\end{abstract}

Summary of a paper, accepted by Phys.Rev.A, March 1994

\section{INTRODUCTION}

Discrete excitations below an ionization limit other than the lowest one leave the atom or ion in autoionizing states. Autoionization is an electron-electron correlation effect involving mixing of discrete configurations with continuous configurations at the same energy. It gives rise to electron emission and to resonance behaviour of the photoabsorption cross section and related quantities such as the angular distribution asymmetry and spin-polarization parameters. The resonance structure can be very complicated as a function of the photon energy. The Fano profile as encountered in low-lying double excitations of He provides a classical example.

The influence of autoionizing states on photoabsorption cross sections is usually so large that it cannot be neglected in any practical applications such as astrophysics and fusion-related plasma physics. Two recent examples illustrate this point. The resonant process

$$
\hbar \omega+3 d^{6} 4 s 5 d \rightarrow 3 d^{6} 4 p 5 d \rightarrow 3 d^{6} 4 s+e^{-}
$$

at $5.4 \mathrm{eV}$ in $\mathrm{Fe}$ has a cross section which peaks at about $10^{4} \mathrm{Mb}$ in association with a dip of about $0.1 \mathrm{Mb}[1]$. The process

$$
\hbar \omega+5 \mathrm{p}^{6} 6 \mathrm{~s} \rightarrow 5 \mathrm{p}^{5} 6 \mathrm{~s} 5 \mathrm{~d} \rightarrow 5 \mathrm{p}^{6}+\mathrm{e}^{-}
$$

at $21.2 \mathrm{eV}$ in $\mathrm{Ba}^{+}$peaks at about $3.10^{3} \mathrm{Mb}$ with a resonance structure which is close to 
Lorentzian[2]. Characteristic of both examples is that the autoionization involves excitation of an electron of the ionic core.

The magnitude of autoionizing structures is not always a decisive factor. It has recently been shown that multielectron excitations involving deep core levels affect the analysis of the $\mathrm{x}$-ray absorption fine-structure in solids[3]. These autoionizing features are rather weak as a recent study of $\mathrm{Kr} 1 \mathrm{snl}$ absorption demonstrates[4]. Nevertheless they cannot be neglected in an accurate fine-structure analysis of solid-state and perhaps molecular photoabsorption spectra. This shows the importance of having access to autoionizing spectra of free atoms since they serve as references for solid-state and molecular effects.

Recent high-resolution measurements[5] of photoabsorption below $\mathrm{N}=2-7$ thresholds of $\mathrm{He}^{+}(\mathrm{N})$ have been compared with hyperspherical close-coupling (HSCC) calculations [6]. The analysis supports the current picture of the doubly-excited He atom as a floppy triatomic molecule with revibrating and stretching modes, approximately described by correlation quantum numbers. The HSCC results seems to be in better agreement with the measurements than multichannel quantum-defect theory (MQDT) fits[5]. A basic question which has remained unanswered is how these autoionization series evolve towards the double-ionization threshold as $\mathrm{N}$ increases. It should be noted that the HSCC method is limited to two-electron systems which illustrates the difficulty of treating autoionization on a universal level.

The random-phase-interaction with exchange (RPAE), which is one of the most successful methods in the theory of photoionization[7], totally fails to predict the width $\Gamma$ and the profile index $q$ of the $\mathrm{n}=4$ resonance [8] in the series

$$
\hbar \omega+3 s^{2} 3 p^{6} \rightarrow 3 s 3 p^{6} n p(n \geq 4) \rightarrow 3 s^{2} 3 p^{5}+e^{-}
$$

of Ar. The interpretation of this series is thus an important test for both $a b$ initio theory and parametrizations. A heuristic inclusion of the Coulomb interaction between the $3 \mathrm{~s}^{2} \mathrm{p}^{6}$ and $3 \mathrm{p}^{4} 3 \mathrm{~d}$ states improves the agreement[8] but the corresponding "two-electron-hole" model still is inconsistent with a proper scattering-theoretical treatment of the process (3). Thus, there is a need for a consistent theory which retains the simplicity of parametrized profiles whether of Fano type[9,10] or of some other type.

\section{EXPERIMENTAL}

Photoion spectra were measured at the Swedish electron storage ring MAX. Using the second order diffraction from a $1 \mathrm{~m}$ normal incidence monochromator total ion yield spectra were recorded with a resolution of $0.4 \AA(20 \mathrm{meV})$ over the autoionization resonances. Figures 1 and 2 show the most important results. We also measured the $\mathrm{Ar}^{+}$photoion spectrum from threshold up to 30 $\mathrm{eV}$. Our result, which is normalized to the incident photon flux, matches Samson's absorption spectrum[11] well except close to $30 \mathrm{eV}$.

Fig. $1 \mathrm{Ar} r^{+}$ion yield
$3 s^{2} 3 p^{6}{ }^{1} S \rightarrow 3 s 3 p^{6} n p{ }^{1} P$
$\rightarrow 3 s^{2} 3 p^{5} \epsilon l^{1} S(l=0,2)$
resonance series measured
with 20 meV resolution
in second order and
normalized to incident
second- order photon flux.

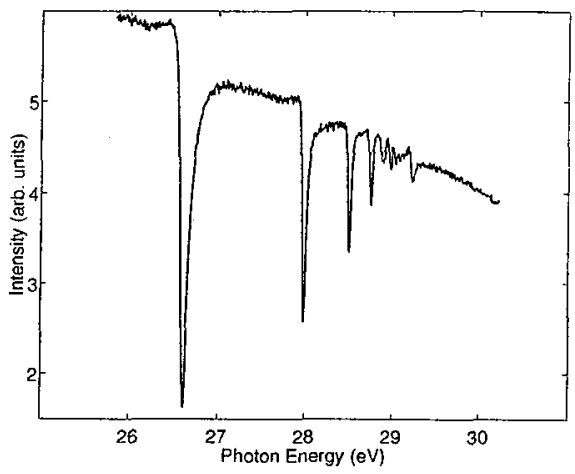


Fig. 2 Ar 3 s resonance fitted to convoluted Fano profile for (a) $n=4$, (b) $n=5$, (c) $n=6$, (d) $n=7$
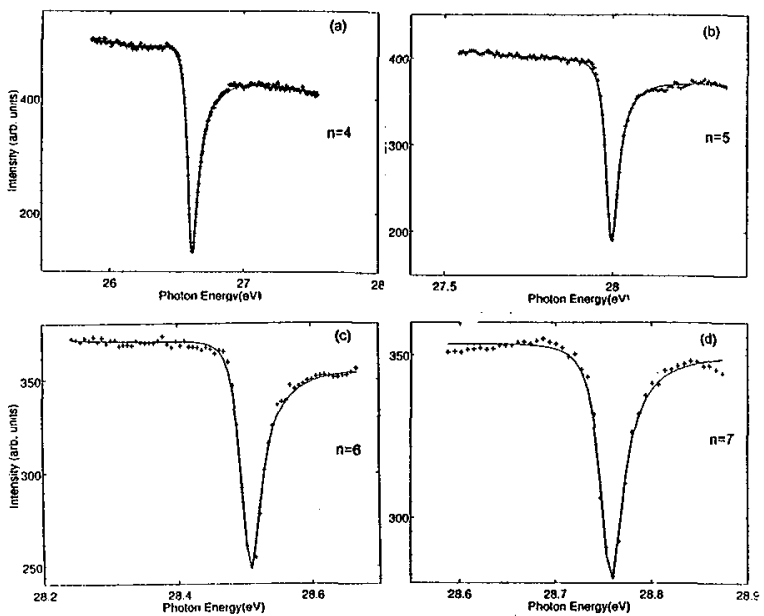

\section{RESULTS AND DISCUSSION}

We have considered the case of $\mathrm{N}_{d}$ discrete states interacting with $\mathrm{N}_{c}$ continua, corresponding to different threshold energies. The residual ions are assumed to be left in definite angular momentum states. The final scattering states of the ion-electron complex thus fulfill the ingoing-wave boundary conditions. As a result of the diagonalization of the discrete-continuous Hamiltonian matrix we obtain a general formula for the transition matrix element between the ground state and the autoionizing out-states. The technique [12] used is a generalization of the Fano-Feshbach formalism. We find that it leads to the Fano-Cooper parametrization [10] of autoionizing resonances for $\mathrm{N}_{d}=1$. We also find that we can retain the form of this formula if a resonance structure is introduced into the continuum wave functions. This structure can be taken to represent discrete adjacent states to the one under scrutiny. Our result is not exact but a generalization of the conclusion of Sánchez and Martín[13] regarding overlapping resonances. The Fano-Cooper parametrization is thus applicable to closely spaced resonan ves with large accuracy. We also show that our formalism leads to the Dubau-Seaton [14] parametrization of entire autoionizing series $\left(\mathrm{N}_{d}\right.$ arbitrary, $\left.\mathrm{N}_{c}=1\right)$ with an exact expression of the closed-channel phase shift.

As Fig.2 indicates we are able to Fano-Cooper parametrize the four first members of the $3 \mathrm{~s} 3 \mathrm{p}^{6} \mathrm{np}(\mathrm{n} \geq 4)$ series and even the fifth $(n=8)$ but with lower statistical accuracy. We thus obtain systematic values of the profile index $q$ and the correlation coefficient $\rho^{2}$ for each resonance in addition to the resonance energies $\mathrm{E}_{r}$ and the widths $\Gamma$. The Dubau-Seaton parametrization was found to be less successful with the exception of $n=4$. This is understandable since it can be shown analytically that Fano-Cooper and Dubau-Seaton approaches should yield similar profiles for isolated resonances.

We have used the multichannel multiconfiguration Dirac-Fock(MMCDF) method[15] to calculate $q, \rho^{2}, \mathrm{E}_{r}$ and $\Gamma$ for $\mathrm{n}=4$. This method is consistent with the time-independent resonant scattering theory[12] which was used to obtain our general results. The calculation does not include the interaction between the $3 \mathrm{~s} 3 \mathrm{p}^{6} \mathrm{np}$ and $3 \mathrm{~s}^{2} 3 \mathrm{p}^{4} 3 \mathrm{dnp}$ states [8] which is the primary correlation effect and treats the final-state channel interaction in an approximate way. The results are encouraging, however, in that all the parameters are of the right order of magnitude and are in accord with the window character of this resonance.

The analysis performed in this work could be applied to other inner-valence autoionization resonance series which have been measured with high resolution. Open-shell atoms and ions would be particularly interesting. The $\mathrm{Cl} 3 \mathrm{~s} 3 \mathrm{p}^{5} \mathrm{np}$ autoionizing series is also of window type with virtually identical quantum defects [16]. The removal of one $3 p$ electron thus does not change the resonance pattern which is very sensitive to electron-electron correlation. The same situation 
prevails for the $\mathrm{Br} 4 \mathrm{~s} 4 \mathrm{p}^{5} \mathrm{np}$ series in comparison with the corresponding series in $\mathrm{Kr}[17]$. One may ask how many $3 p$ or $4 p$ electrons must be removed before the resonance character changes. The answer would unambiguously reveal the origin of the low $q$ value. The Ga $4 \operatorname{sipnp}(\mathrm{n}=5,6)$ resonances are definitely not of window type although they are asymmetric [18]. Systematic studies of this kind in association with large-scale MMCDF computations could reveal the inherent mechanisms behind the shape of the resonances.

\section{References}

[1]Sawey, P.M.J. and Berrington, K.A., J.Phys.B23 (1990) L817

[2]Lyon, I.C., Peart, B. and Dolder, K., J.Phys. B20 (1987) 1925

[3]Li, G., Bridges, F. and Brown, G.S., Phys.Rev.Lett. 68(1992) 1609

[4]Schaphorst, S.J. et al, Phys.Rev.A47(1993)1953

[5]Domke, M. et al, Phys.Rev.Lett. 66(1991)1306

[6]Tang, J.-Z., et al, Phys.Rev.Lett 69(1992) 1633

[7]Amusia, M.Ya, Atomic Photoeffect (Plenum, New York 1990)

[8]Amusia, M.Ya. and Kheifets, A.S., Phys.Lett.82A(1981)407

[9]Fano, U., Phys.Rev.124(1961)1866

[10]Fano, U. and Cooper, J.W., Phys.Rev. A137(1965)1364

[11]Samson, J.A.R., Adv.At.Mol.Phys.2(1966)177

[12] Åberg, T., Physica Scripta 21(1980)495

[13]Sánchez, I. and Martín, F., Phys.Rev.A44(1991)7318

[14]Dubau, J. and Seaton, M., J.Phys.B17(1984)381

[15]Tulkki, J., Åberg, T., Mäntykenttä, A. and Aksela, H., Phys.Rev.A46(1992)1357

[16]van der Meulen, P., Krause, M.O., Caldwell, C.D., Whitfield, S.B. and de Lange, C.A., J.Phys.B24(1991)L573; Robicheaux, F. and Greene, C.H., Phys.Rev.A47(1993)1066

[17]van der Meulen, P., Krause, M.O. and de Lange, C., J.Phys.B25(1992)97

[18]Caldwell, C.D., Krause, M.O. and Jíménez-Mier, J., Phys.Rev.A37(1988)2408 